\title{
Chronic middle foot pain in a patient with a swollen leg
}

\author{
Grzegorz Marek Karwacki • Ueli Studler • \\ Tilo Niemann
}

Received: 10 May 2011 /Revised: 29 June 2011 / Accepted: 4 July 2011 / Published online: 29 July 2011

(C) ISS 2011

\section{Question}

A 32-year-old male patient with a known congenital syndrome was referred to our radiology department for work-up of persistent worsening foot pain over 4 weeks. No history of previous trauma was identified. Radiography and (for further work-up) MRI and CT examinations were performed (Figs. 1, 2, 3).

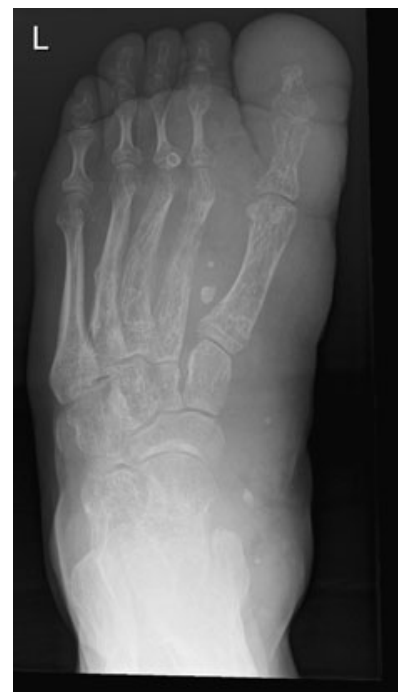

Fig. 1 DP radiograph of the left foot

The diagnosis can be found at doi:10.1007/s00256-011-1238-8

G. M. Karwacki $(\bowtie) \cdot$ U. Studler $\cdot$ T. Niemann

Department of Radiology, University Hospital Basel,

Petersgraben 4,

4031 Basel, Switzerland

e-mail: karwackig@uhbs.ch

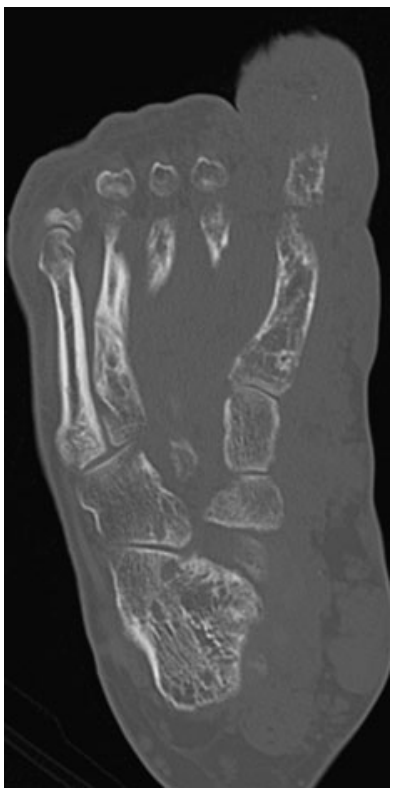

Fig. 2 Axial MR T1-weighted fat-saturated post contrast image of the

left foot

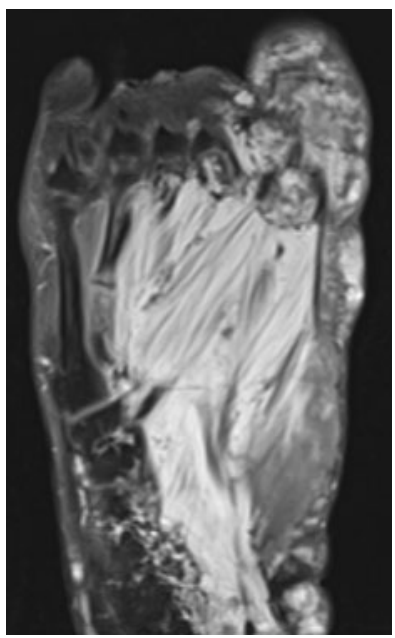

Fig. 3 CT, axial reformation of the left foot 